\title{
La formación permanente de los asesores técnico-pedagógicos en México: una propuesta de criterios de formación
}

\author{
The continuous training of technical-pedagogical \\ advisors in Mexico: a proposal training criteria
}

Lissete Huerta Dominguez y Javier Onrubia Goñi*

\begin{abstract}
For several decades, school consultation has being considered as an essential factor in order to improve the quality of schools in Mexico. Therefore, debating about the role of school consultants and how these professionals should be trained in specific competences, it has become an essential issue. This article focuses in presents a model of collaborative consultation, which from our perspective, gives consultants a theoretical and methodological reference, that allows them to assist teachers and students to learn better. Based on this model, we highlight some competences that consultants should master to develop this type of consultation, and give some methodological criteria for designing processes of continuous training towards to promote the development of these competencies in order to contribute to its professional growth.
\end{abstract}

Key words: advisors training, collaborative consultation, professional skills

to colaborativo, competencias profesionales

\footnotetext{
*Departamento de Psicología Evolutiva y de la Educación de la Universidad de Barcelona (España); lisshuerta@gmail.com; javier.onrubia@ub.edu.
} 


\section{LA RELEVANCIA DE INTRODUCIR UN MODELO DE ASESORAMIENTO COLABORATIVO PARA LA MEJORA DE LA EDUCACIÓN MEXICANA}

A lo largo de los últimos 30 años, la educación en México ha vivido un estado de permanentes reformas (Tapia, 2008) dirigidas a elevar, de alguna u otra manera, la calidad de la educación que se brinda en las escuelas de educación básica. Esto ha implicado, para el Sistema Educativo Nacional, el desarrollo de acciones y el uso u optimización de recursos económicos, materiales y humanos en distintos momentos y espacios.

En su reflexión acerca de la manera en que se han llevado a cabo las reformas educativas en México, Cordero, Fragoza y Vázquez (2015) y Tapia (2008) destacan que la estrategia más empleada para la transmisión del enfoque y los preceptos de cada una de las reformas educativas es la denominada "capacitación en cascada". Esta estrategia consiste en entregar la información que se desprende de la reforma educativa por niveles: un primer nivel vinculado al trabajo que hacen las mesas técnicas estatales (que se crean para esta tarea) con los supervisores escolares; un segundo nivel, que remite a la transmisión de los contenidos de los supervisores escolares a los directores de escuela y, finalmente, un tercer nivel, donde estas últimas figuras transmiten la información a los profesores de cada centro educativo. El objetivo de esta estrategia es que los profesores adquieran y, posteriormente, introduzcan a sus prácticas los lineamientos, preceptos y reglas de operación que rigen una reforma educativa.

Este modelo de asesoramiento, esencialmente informativo y jerárquico, basado en la entrega de la información y en la transmisión de consignas de la autoridad a los profesores presenta, desde nuestro punto de vista, algunas limitaciones. La fundamental es que corre un riesgo considerable de no lograr transformar las prácticas educativas (Gutkin, 1999). Como afirma Fullan (2002), los cambios e innovaciones que son propuestos simplemente desde fuera de los centros escolares, difícilmente son adoptados como propios por los usuarios (profesores, directores, padres de familia), originando que en muchas ocasiones estas se difuminen y nunca lleguen a implementarse. Estas ideas nos parecen potentes 
y nos permiten pensar que la propuesta que queremos plantear al pugnar por un tipo de asesoramiento alternativo, colaborativo entre los profesores y el asesor tiene sentido para contribuir a los objetivos de cambio y mejora de la enseñanza que pretenden, en último término, las reformas educativas.

Proponer la adopción de un modelo de asesoramiento colaborativo frente a un modelo de asesoramiento prescriptivo y jerárquico resulta, por tanto y a nuestro juicio, coherente con las propuestas de cambio y mejora que hace la reforma educativa vigente (2013). La reforma educativa plantea, en efecto, 14 transformaciones principales, las cuales contribuirán a mejorar la calidad educativa de nuestro país (en términos de autonomía de la gestión escolar, formación continua profesional, fortalecimiento de la equidad y la inclusión). Desde nuestra opinión, optar por un proceso de asesoramiento colaborativo que recoja las necesidades de las escuelas, donde se permita a los profesores convertirse en agentes activos del cambio negociando y no imponiendo las innovaciones a introducir, brindándoles acompañamiento a lo largo del proceso y al mismo tiempo ayudándolos a valorar la mejoras introducidas durante el camino recorrido y al final de este, contribuirá a lograr cambios más significativos y más duraderos en sus prácticas impulsando desde la escuela esas transformaciones que nuestro sistema educativo necesita.

El tipo de asesoramiento que proponemos tiene unas características específicas y demanda de quien lo desarrolle un proceso de formación centrado en la adquisición de ciertas competencias profesionales. Creemos que, aunque no es tarea fácil, no es una tarea imposible, ya que, como Tapia menciona (2008), la figura del asesor en México está en proceso de construcción. Considerando esta idea, en este artículo nos interesa presentar algunos planteamientos que puedan apoyar la mejora de los procesos formativos de estos agentes educativos con la intención de apoyar la transición de un asesoramiento meramente centrado en la transmisión de contenidos a un asesoramiento de proceso, donde los profesores sean agentes activos de su desarrollo y crecimiento profesional. 


\section{PROPUESTA DE UN MODELO DE ASESORAMIENTO COLABORATIVO Y CENTRADO EN LA MEJORA DE LAS PRÁCTICAS EDUCATIVAS}

Una vez abordada la relevancia de un modelo de asesoramiento colaborativo como estrategia para mejorar la educación en nuestro país, intentaremos detallar sus características. Un primer acercamiento a esta descripción es destacar que este tipo de asesoramiento centra su de atención en la escuela, es decir, las ayudas que se prestan se dirigen a contribuir a que los profesores implementen mejoras en su práctica pedagógica. Para que este tipo de asesoramiento se pueda desarrollar, es indispensable que las mejoras que se planteen introducir sean consensuadas y aceptadas por los profesores. Estos deben, por un lado, querer esos cambios (considerarlos deseables), y por otro, considerar que pueden llevarlos a cabo (considerarlos factibles). Al mismo tiempo, deben percibir que es un trabajo que se realiza en conjunto, donde cada uno de los participantes se sienta reconocido y valorado, capaz de aportar conocimientos, ideas y puntos de vista para lograr el cambio. Este tipo de asesoramiento no solo se convierte en un brindar soluciones al instante a los problemas, sino que se considera como una herramienta capaz de incentivar e instaurar cambios a largo plazo.

En los siguientes párrafos intentaremos caracterizar este tipo de asesoramiento "colaborativo y centrado en la mejora", al mismo tiempo que introduciremos algunas anotaciones del porqué consideramos que puede ser la mejor alternativa para alcanzar los logros educativos que se le han planteado a estos agentes educativos en México. Las propuestas de distintos autores (Bassedas, 1998; De la Oliva, Martín y Velaz de Medrano, 2005; Gutkin, 1999; Monereo y Solé, 1996; Rosenfield, 2008; Shulte y Osborne, 2003; Solé, 1998) nos permitirán sustentar con mayor fuerza lo que nos interesa plantear como propuesta de innovación en el ámbito de la asesoría en México. 
Para iniciar esta caracterización decidimos retomar las aportaciones que Solé (1998) hace sobre los elementos básicos que definen un asesoramiento de este tipo. Estos puntos son:

1. El trabajo conjunto a lo largo del proceso.

2. La responsabilidad compartida.

3. La construcción de significados compartidos entre asesor y asesorados.

Cuando nos referimos a un trabajo conjunto a lo largo del proceso, estamos hablando de lo que autores como García, Rosales y Sánchez (2003), Rosenfield (2008) y Solé (1998) mencionan al reconocer que, para que este proceso se dé, cada cual (asesor y asesorados) debe participar desde su formación peculiar y aportar sus conocimientos, vivencias y puntos de vista para el logro de objetivos compartidos (diseńo y desarrollo de procesos de enseñanza que ayuden al alumno a avanzar en su aprendizaje). El reconocimiento de lo que uno y el otro pueden aportar al proceso y la negociación de los objetivos y tareas se convierte en un rasgo característico de este elemento (Lago y Onrubia, 2008). El trabajo conjunto implica, entonces, que tanto el asesor como el asesorado consensen y lleguen a acuerdos de lo que esperan alcanzar y, en consecuencia, de lo que corresponde hacer a cada uno para lograrlo. El asesor, en este sentido, deber ser muy cuidadoso y no imponer sus ideas o puntos de vista, lo cual no quiere decir que deba dejar el proceso al azar, sino que debe tener claro hacia dónde dirigir a los asesorados (Gutkin, 1999).

En cuanto a la responsabilidad compartida, autores como García, Rosales y Sánchez (2003), Solé (1998) y Lago y Onrubia $(2008,2011)$ señalan la necesidad de que tanto el asesor como el asesorado se responsabilicen de las tareas que implica la resolución conjunta de problemas y la mejora de las prácticas. Esto significa que, para la consecución del objetivo planteado, ambas partes (asesor y profesores) deberán asumir la responsabilidad global sobre el proceso y la responsabilidad específica de realizar las tareas que les correspondan. Esta idea plantea ir traspasando esta responsabilidad a los profesores; así, de manera gradual, el asesor podrá ir retirando su participación para que los asesorados 
puedan asumirla de manera plena. Clement, Di Napoli, Gilis, Buelens y Frenay (2011) y Swefield (2008) proponen que el asesor que desarrolle este tipo de tareas lo haga desde una postura particular, actuando como un "amigo crítico" (critical friend) que provoque la responsabilidad de los profesores a través de la reflexión sobre su práctica, aportando otro punto de vista y proponiendo elementos críticos y retos abordables, todo esto en un clima de confianza y seguridad. Para estos autores, este clima de confianza es la base de estos intercambios y de la generación de propuestas de cambio. Este rasgo característico de la colaboración permitirá a los asesorados reconocer su capacidad de resolución de problemas y de mejora de las prácticas, y asumir poco a poco de manera autónoma el control del proceso. Esto les permitirá sentirse capaces de actuar ellos solos en otras situaciones similares.

Por último, y con relación a la construcción de significados compartidos entre asesor y asesorados, autores como Solé y Martín (2011) y Hylander (2012) subrayan que en un asesoramiento colaborativo es posible ir acercando posiciones, compartiendo significados y, de manera paralela, modificando concepciones, expectativas y conocimientos previos. Esto nos lleva a pensar que, en un proceso de colaboración, el cambio en las ideas y concepciones de los profesores se dará en la medida en que se cuestionen sus representaciones anteriores y se les permita la adopción de otras más potentes. Esto debe darse, sin lugar a dudas, en un proceso de negociación y puesta en común de esas ideas, donde las propias de cada uno y las nuevas se reconozcan como valiosas y necesarias para la consecución de los cambios a promover.

Tal y como lo estamos caracterizando, este proceso de asesoramiento colaborativo presenta dos dimensiones particulares, interrelacionadas pero distintas. Por un lado, una dimensión relacionada con la construcción de los aspectos referidos al proceso de resolución conjunta del problema, y por otro, una dimensión enfocada a la parte afectiva y emocional, relacionada más con la construcción de la colaboración dentro del proceso. Muchos autores reconocen que la existencia y equilibrio entre estas dos dimensiones es clave para el desarrollo de procesos de asesoramiento más eficaces (Clement, Di Napoli, Gilis, Buelens y Frenay, 2011; Garbacz, Woods, Swanger-Gagné, Taylor y Black, 
2008; Rosenfield, 2008; Sánchez y García 2011; Swefield, 2008; Thompson, 2013). Estas dos dimensiones, que de ahora en adelante denominaremos como las caras "fría" y "cálida" del proceso de asesoramiento colaborativo (Sánchez y García 2011), las abordaremos de manera breve para terminar de caracterizar lo que un proceso de asesoramiento colaborativo implica.

En cuanto a la cara "fría" del proceso de asesoramiento, a lo largo de la descripción del asesoramiento colaborativo que hemos realizado, la resolución conjunta de un problema aparece como el escenario principal del proceso de colaboración; es decir, es siempre en este contexto (resolver un problema y conseguir un cambio juntos) en el que un proceso de asesoramiento colaborativo tiene sentido. Sánchez (2000) presenta un primer acercamiento a esta cara del proceso de asesoramiento definiéndola, explícitamente, como un proceso de resolución conjunta de problemas. Este proceso tiene un componente esencialmente cognitivo, ya que es donde se construye una representación mental conjunta de a dónde se desea llegar y se plantean las acciones que ayudarán a alcanzar el estado deseado.

Para este autor (Sánchez 2000, Sánchez y García, 2011), así como para otros tantos (Lago y Onrubia 2011; Rosenfield, 2008) esta cara del proceso de asesoramiento se concreta a través de un conjunto de fases. Si bien la presentación exhaustiva de ellas no es el tema de este artículo, la mayoría de autores identifican algunos momentos comunes de especial importancia para el éxito del proceso. El primero es un momento de análisis, comprensión y delimitación del problema, en que asesor y asesorados discuten y circunscriben, de común acuerdo, el problema que intentarán resolver. El segundo es un momento en que se analizan conjuntamente las prácticas en curso y se diseñan, también en mancomún, las propuestas de mejora que se creen capaces de introducir. El tercero remite a la implementación de las mejoras y al acompańamiento, seguimiento y valoración de la puesta en marcha de las mismas. Finalmente, un cuarto momento se dedica a la evaluación del asesoramiento, incluyendo la valoración de las mejoras que se pretendía introducir, de los roles que cada uno desempeñó y del proceso mismo de asesoramiento. 
En cuanto a la cara "cálida" del asesoramiento, como mencionábamos en párrafos anteriores, está relacionada con los componentes emocionales y motivacionales implicados en desarrollar grupalmente acciones para resolver problemas. Estos componentes se asumen como inherentes a las personas que enfrentan problemas y quieren resolverlos. Al mismo tiempo, se reconoce la influencia del asesor para poder establecer un clima de confianza y seguridad que permita a los asesorados sentirse escuchados, valorados y reconocidos a lo largo del proceso de asesoramiento.

Autores como Lago y Onrubia (2011), Rosenfield (2008), Sánchez (2000), Sánchez y García (2011) destacan que, para que se dé una relación de colaboración a lo largo de todo proceso de asesoramiento es necesario que se creen las condiciones necesarias. Estas condiciones tienen que ver con la creación de un clima de aceptación, valoración y confianza, que provoque en los asesorados expresar sus ideas, concepciones y formas de ver el problema. La creación de este clima de confianza asegurará que los profesores implicados en los procesos de asesoramiento se sientan involucrados en las mejoras. Para ello, el asesor tiene que transmitir a los profesores que: a) se les ha escuchado, b) que se han comprendido sus necesidades y c) que se han valorado los recursos con los que cuenta, así como los intentos que hace por resolver el problema. Por otro lado, el asesor debe de utilizar una serie de recursos que le permitan mantener ese clima de confianza (habilidades de comunicación, definir el problema en términos de comportamiento, habilidades de escucha), así como poseer ciertas características (escuchar, ser asertivo, honesto y crítico) que permitan a los otros establecer espacios de comunicación abierta (Clement et al., 2011; Swefield, 2008).

\section{NECESIDADES FORMATIVAS PARA DESARROLLAR UN ASESORAMIENTO COLABORATIVO Y CENTRADO EN LA MEJORA}

Una vez explicitado el marco general bajo el cual nos situamos para considerar la implementación de procesos de asesoramiento colaborativo como un modelo de intervención encaminado a la mejora de las prácticas educativas, introduciremos en este aparta- 
do los conocimientos, habilidades y actitudes que un asesor debe poseer y desarrollar para estar en condiciones de implementar este tipo de asesoramiento. Es importante destacar que lo que a continuación presentamos está encaminado a perfilar a un tipo de asesor, un asesor interesado en desarrollar procesos de asesoramiento colaborativo y encaminado a la transformación de las prácticas educativas. No nos interesa, por tanto, la formación de asesores en toda su extensión y complejidad, ni por ello nos es útil presentar listados completos de competencias que algunos autores interesados en esta tarea proponen. Nos interesa de manera específica indicar aquellas que, de acuerdo con lo expuesto, pueden ayudar a formar al asesor técnico-pedagógico en el núcleo de elementos necesarios para desarrollar procesos de asesoramiento colaborativo dirigidos a la mejora de las prácticas de enseñanza.

La propuesta que planteamos a continuación se apoya en la revisión de trabajos de diversos autores (Luque, 2005; Mauri, 1996; Solé, 2005; Vélaz de Medrano, Moya, López-Martín y Manzano-Soto, 2013; West, Idol y Cannon, 1988) y trata de integrarlas en una propuesta de conjunto de las competencias que, desde nuestra concepción de asesoramiento y del tipo de relación que se debe establecer, son necesarias para apoyar las prácticas de los asesores técnico-pedagógicos que decidan implementar procesos de asesoramientos colaborativos. A su vez, consideramos que esta propuesta puede servir de base para la identificación de las necesidades de formación de estos agentes educativos. Para su presentación en este artículo hemos optado por dividir estas competencias, relacionándolas con las dos caras del proceso de asesoramiento colaborativo que acabamos de presentar. Es necesario destacar que estas competencias, si bien analíticamente son distintas, no pueden ni deben enseñarse por separado, por lo que cuando se piensen y desarrollen procesos de formación de estas figuras, las sesiones y las actividades deberán estar dirigidas a lograr su aprendizaje conjunto. 


\section{Competencias que favorecen la resolución conjunta de problemas}

1. Guiar en la resolución de problemas, conociendo sobre las diversas fases que conforman el proceso de asesoramiento para ajustarlas en las situaciones y contextos, de acuerdo con las necesidades de asesoramiento específicas.

2. Ayudar a reflexionar a los asesorados sobre los procesos de cambio y mejora que pueden instrumentar, de manera que con ello puedan construir o enriquecer representaciones más completas y compartidas de esas innovaciones.

3. Valorar alternativas de solución de problemas anticipando posibles consecuencias antes de proponer las acciones a los asesorados, y ayudando a estos últimos a encontrar la mejor solución en su contexto al problema planteado como contenido de mejora.

4. Gestionar los recursos, espacios y tiempos para desarrollar el proceso de asesoría de manera eficaz.

5. Gestionar la temporización de las actividades del proceso de asesoramiento para facilitar la toma de decisiones entre el asesor y el asesorado en cada fase del proceso.

6. Desarrollar una variedad de técnicas para recopilar información, que permitan identificar y clarificar el problema detectado como contenido de mejora.

7. Analizar y diseñar de manera conjunta los cambios que deberán introducirse para lograr los objetivos planteados en el proceso de asesoramiento, asegurando que se llevan efectivamente a la práctica.

8. Rediseńar, mantener o interrumpir las intervenciones a partir de la evaluación que se realice durante el periodo de implementación de las mejoras.

9. Valorar los puntos débiles y fuertes del proceso de asesoramiento para modificar las actuaciones de los participantes que favorezcan el mejor desarrollo del proceso de asesoramiento.

10. Ayudar a generar estos cambios en la práctica de manera permanente, para que los docentes y la escuela puedan sostenerla en el tiempo de manera autónoma. 


\section{Competencias que favorecen la construcción de la relación de colaboración}

1. Establecer y mantener una relación cordial, respetuosa, congruente y empática, propiciando interacciones donde los asesorados se sientan escuchados y valorados.

2. Comunicar de forma clara y eficiente las ideas, aclaraciones, preguntas... para evitar sesgos en la comunicación con los asesorados, facilitando así los intercambios comunicativos.

3. Generar confianza en los espacios de asesoramiento para que los asesorados puedan expresar sus ideas, opiniones, puntos de vista y formas de entender el proceso de asesoramiento, facilitando igualdad de oportunidades para hacerlo.

4. Asegurar la implicación de los asesorados durante todo el desarrollo del proceso de asesoramiento.

5. Apoyar la resolución de las dificultades que surjan durante el proceso de diseño e instrumentación de los cambios, ayudando a entender estas dificultades como parte habitual del proceso.

6. Utilizar los recursos discursivos necesarios que faciliten el proceso de colaboración.

7. Manejar la viabilidad y la deseabilidad de la puesta en marcha de las propuestas de mejora, para estar en condiciones de ajustar sus actuaciones y mantener la motivación en el proceso en el que los asesorados están implicados.

8. Evitar la dependencia del asesor a lo largo, y especialmente al final, del proceso, promoviendo la autonomía, capacitación y empoderamiento progresivos de los docentes, individual y colectivamente.

Como podemos darnos cuenta, las competencias que proponemos como perfil del asesor, desde este marco, se vinculan estrechamente con la prioridad de establecer un proceso de asesoramiento centrado en las necesidades de los asesorados. Este tipo de proceso de asesoramiento reconoce, respeta y considera los puntos de vista y formas de representación de los problemas como elementos importantes que contribuirán a la construcción conjunta de las mejoras y, al mismo tiempo, reconoce que el ase- 
sor debe ser un experto de procesos que puedan guiar la concreción y puesta en práctica de estas mejoras.

\section{CRITERIOS METODOLÓGICOS PARA PENSAR PROCESOS DE FORMACIÓN PERMANENTE QUE CONTRIBUYA A DESARROLLAR PROCESOS DE ASESORAMIENTO COLABORATIVOS Y CENTRADOS EN LA MEJORA}

Como lo mencionamos a lo largo del recorrido anterior, los criterios metodológicos que a continuación se presentan fueron seleccionados bajo la misma lógica de las competencias arriba expuestas; es decir, pensando en procesos de formación permanente que permitan a los asesores desarrollar procesos de asesoramiento colaborativos. Estos criterios, por tanto, buscan generar las condiciones óptimas para que las competencias que se pretenden promover puedan ser aprendidas y desarrolladas.

\section{Dotar de un marco teórico de referencia que} permita a los asesores comprender los procesos de enseñanza y aprendizaje desde una perspectiva constructivista y, en consecuencia, reconocer la importancia de adoptar un modelo de asesoramiento que responda a esos planteamientos

Las aportaciones de Luque (2005), Mauri (1996) y Solé (2005), así como las de Truscott, Krekey, Bolling, Psimas, Graybill, Albritton y Schwartz (2012), acerca de la importancia de contar con una teoría de base que sustente las intervenciones que realiza el asesor, nos permiten considerar este criterio como parte fundamental de cualquier programa de formación permanente que pretenda desarrollar las competencias antes mencionadas. El hecho de comprender las premisas y planteamientos que sustentan la implantación de procesos de asesoramiento colaborativos y centrados en la mejora permitirá que las intervenciones de los asesores se dirijan a ayudar a los agentes educativos y, a la institución en su conjunto, a mejorar la calidad de los procesos de enseñanza y aprendizaje desde una perspectiva constructivista. Este criterio nos lleva a considerar que, en los programas de formación permanente que se diseñen, deberán incluirse de manera pun- 
tual actividades que permitan trabajar algunos principios teóricos básicos del modelo que sustenta el actuar de los asesores. A este respecto, las actividades de discusión entre los participantes y el formador deberán promover la contrastación de estos contenidos con su práctica asesora. Consideramos que, si estas actividades se desarrollan de manera eficaz, pueden ayudar a los participantes a comprender e interiorizar los conceptos y preceptos del asesoramiento colaborativo.

\section{Tomar como proceso transversal de la formación la reflexión sobre la práctica, elemento fundamental para el desarrollo de las competencias profesionales necesarias y de la profesionalización de estos sujetos}

La importancia atribuida a la reflexión sobre la práctica en la formación de los asesores por muchos de los autores que hemos revisado (Mauri, 1996; Solé, 2005), así como su consideración más amplia como parte fundamental de la profesionalización de los agentes involucrados en el ámbito educativo (Perrenoud, 2004; Schön, 1998), nos permite justificar la introducción de este criterio como elemento indispensables en cualquier proceso de formación de esta índole. Por tanto, durante estos procesos formativos, el desarrollo de actividades donde los asesores participen activamente en la reflexión y análisis de sus experiencias, se convierte en un dispositivo provocador de desequilibrio y equilibrio de sus ideas, concepciones y forma de valorar su práctica. Este tipo de actividades provocarán que los participantes reflexionen sobre su quehacer considerando tres cuestiones importantes: 1) las características mismas del contexto en el que intervienen, 2) identificación de los problemas reales a los que se han enfrentado y pueden enfrentarse y 3) el planteamiento de respuestas a estas situaciones problemáticas que se producen cuando se producen. La propuesta de ejes de reflexión que guíen los comentarios, así como la discusión entre pares y el trabajo de reflexión conjunta sobre la práctica son algunos de los elementos que, a nuestro juicio, pueden ayudar a concretar este criterio. 


\section{Considerar el contexto en el que desenvuelven su tarea asesora como objeto de análisis, para así responder a necesidades reales que enfrentan en su cotidianeidad}

Entendemos que la consideración del contexto de trabajo de los docentes es una parte indispensable dentro de la formación de asesores desde la perspectiva que nos compete. Este criterio nos lleva a vislumbrar que el tipo de formación que se propone a los asesores les plantee trabajar con contenidos de mejora (Lago y Onrubia, 2008) auténticos y contextualizados, es decir, que puedan, por ejemplo, diseñar planes de intervención considerando una necesidad real de un grupo real de profesores. Esto les provocará centrar su análisis, reflexión y futura intervención en situaciones concretas. Consideramos que, si la formación toma en cuenta este criterio, los aprendizajes adquiridos por los asesores durante el proceso de formación serán más significativos y tendrán mayor sentido para su intervención.

\section{Desarrollar situaciones de práctica y simulación, empleando distintos recursos (análisis de casos, análisis de prácticas, role playing)}

Diversos autores que aportan criterios relevantes para la construcción de programas de formación dirigidos a asesores que desean desarrollar procesos de asesoramiento colaborativos destacan la necesidad de desarrollar situaciones de práctica y simulación (Showers y Joyce 1996) que promuevan el desarrollo de habilidades que, posteriormente, puedan ser aplicadas en la práctica (Reschly, 1993; Pérez Cabaní y Carretero, 2009). Por esta razón, nos atrevemos a proponer que los programas de formación incluyan actividades que induzcan a los asesores a poner en juego estas habilidades en situaciones que se parecen a, o son tomadas de, la realidad en donde asesoran. Hacer esto implicaría que dentro del programa de formación, se procurara implementar actividades de simulación. En algunos casos, y dependiendo de lo que el asesor formador identifique, estas actividades podrán desarrollarse a través del análisis de algunas situaciones didácticas o realizando 
LA FORMACIÓN PERMANENTE DE LOS ASESORES TÉCNICO PEDAGÓGICOS EN MÉXICO...

algunos ejercicios de role playing y/o plasmando sus reflexiones a través de la escritura.

\section{Dotar a los asesores de instrumentos prácticos que les permitan concretar y dar seguimiento a los cambios introducidos a la práctica docente}

Los instrumentos de recopilación de información, el procedimiento para poder llevar a cabo esta recopilación, así como una valoración adecuada y fundamentada sobre las necesidades de asesoría integran un ciclo indispensable para el inicio de un proceso de asesoramiento que pretenda innovar y mejorar las prácticas educativas. Contar con esta valoración, construida con la integración de información de diversas fuentes (documentos, entrevistas, diálogo con los profesores, etc.) y con la participación de todos los agentes involucrados (equipo directivo, profesores) permite al asesor pensar y ajustar las ayudas que prestará a lo largo del proceso de asesoramiento que desarrolle. Este criterio nos lleva a pensar en la necesidad de que los programas de formación que se diseñen cuenten con espacios donde los participantes puedan pensar y construir aquellos instrumentos de recopilación de información, y de concreción y seguimiento de los cambios introducidos en la práctica docente que consideren necesarios y pertinentes para esta tarea.

\section{Contemplar acciones de seguimiento a la práctica asesora para garantizar la transferencia de las competencias adquiridas durante la formación hacia la práctica}

Una de las premisas que consideramos que debe sustentar la construcción de programas de formación dirigidos a formar asesores competentes para desarrollar procesos de asesoramiento colaborativo es la relevancia del acompańamiento y el seguimiento al trabajo real de los asesores. Gravios, Knotek y Babinsky (2002) muestran que, si no existe este elemento dentro de la formación, la aplicación de las habilidades y conocimientos adquiridos puede no llevarse a cabo en la práctica. En ese sentido, los programas de formación deberían incorporar sesiones de seguimiento y acom- 
pañamiento al trabajo que los asesores realizan con los docentes. Consideramos que estas sesiones de seguimiento comprometerán una doble finalidad: por un lado, ayudar a la aplicación de las habilidades adquiridas en el proceso de formación, y por otro, valorar las dificultades, los logros y las acciones de mejora que deben introducirse en las intervenciones de los asesores. Estas sesiones de seguimiento se distinguen como un proceso de retroalimentación, ya que el formador tendrá la responsabilidad de devolver información acerca de lo que los asesores construyan y compartan antes, durante y después de estas sesiones de seguimiento.

\section{Crear espacios de intercambio con otros asesores, que les permitan analizar y valorar sus intervenciones para introducir cambios en su práctica}

El trabajo con otros y las aportaciones que se pueden dar y recibir de los otros se convierte en un elemento representativo de una visión social y constructivista de los procesos de aprendizaje, por lo que un programa de formación de esta índole, debe tomar como fuente de aprendizaje este tipo de interacciones entre los participantes. Considerando lo anterior, proponemos que el trabajo colaborativo se convierta en una herramienta imprescindible para el desarrollo de las competencias planteadas con anterioridad. Este criterio nos lleva a pensar que los asesores deberán compartir y discutir situaciones reales de su tarea asesora.

\section{Procurar que en el proceso de formación se propicie la cesión de control por parte del formador a los asesores participantes}

La cesión del control (Castello y Monereo, 2005), como elemento clave de cualquier proceso formativo, se convierte en un criterio indispensable en el diseño de programas de formación dirigidos a constituir un asesor colaborativo. Para ello, se propone diseñar una estructura en que, al inicio del proceso de formación, el asesor formador sea quien "guíe" fundamentalmente las actividades, siendo el encargado de presentar los contenidos que se van a desarrollar, y el responsable de regular el proceso conjunto. Posteriormente, conforme avance el proceso, se propone realizar 
un cambio en la estructura de las sesiones, que ceda en mayor medida a los participantes el control de su proceso de formación y aumente su protagonismo para pensar, diseñar, implementar y valorar actuaciones y procesos de asesoramiento.

En conjunto, consideramos que estos criterios metodológicos pueden contribuir de manera positiva a la formación de los asesores técnicos de nuestro país. Sabemos que la integración de los criterios anteriormente expuestos a un sistema de formación permanente de asesores implica transformar la visión que se tiene del asesor y en consecuencia del tipo de asesoramiento que este debe brindar, y reconocemos que representa un cambio a largo plazo que involucra una serie de fases y diversos niveles de actuación, y que requerirá el esfuerzo de muchos agentes. Al mismo tiempo, confiamos en que la atención que se le pueda dar a estos aportes contribuirá a mejorar la formación de los asesores en México y en consecuencia impactará de manera positiva en la introducción de mejoras en las prácticas de cada aula de nuestras instituciones educativas.

\section{REFERENCIAS BIBLIOGRÁFICAS}

Bassedas, E. El asesoramiento psicopedagógico: una perspectiva constructivista, Cuadernos de pedagogía, 1988, 159, 65-71.

Castelló, M. y Monereo, C. Análisis de contextos de asesoramiento. Ayudando a redescribir la representación de los problemas, en C. Monereo y J. I. Pozo (Eds.). La práctica del asesoramiento educativo a examen, Barcelona, Graó, 2005, pp. 323-340.

Clement, R., Buelens, H., Di Napoli, R., Frenay y M. Gilis, A. "Educational Consultation for reflective-dialogic partnerships: a possible model", en Recherche and Formation, 67, 2011, pp. 31-48.

Cordero, G., Fragoza, A. y Vázquez, M. A. "El Servicio de Asistencia Técnica a la Escuela: aproximaciones a su configuración en México", en Revista Electrónica de Investigación Educativa, 17(1), 2015, pp. 55-71. Disponible en http://redie. uabc.mx/vol17no1/contenido-cordero-fragozav.html 
De La Oliva, D., Martín, E., y Vélaz De Medrano, C. “Modelos de intervención psicopedagógica en centros de educación secundaria: identificación y evaluación”, en Infancia y Aprendizaje, 28 (2), 2005, pp. 115-139.

Erchul, W. P. "On dominance, cooperation, teamwork, and collaboration in school-based consultation", en Journal of Educational and Psychological Consultation, 3, 1992, pp. 363-366.

Fullan, M. "El significado del cambio educativo: un cuarto de siglo de aprendizaje", en Profesorado, revista de curriculum y formación del profesorado, 6 (1-2), 2002, pp. 1-14.

Garbacz, S. A., Woods, K. E., Swanger-Gagné, M. S., Taylor, A. M., Black, K. A., y Sheridan, S. M. "The effectiveness of a partnership-centered approach in conjoint behavioral consultation", en School Psychology Quarterly, 23(3), 2008, 313.

García, J. R., Rosales, J., y Sánchez, E. "El asesoramiento psicopedagógico como construcción de significados compartidos: un estudio sobre su dificultad", en Cultura y Educación, 5 (2), 2003, pp. 129-148

Gravios, T. A., Knotek, S., \& Babinski, L. "Educating practitioners as consultants: Development and implementation of the instructional consultation team consortium", en Journal of Educational and Psychological Consultation, 13(1-2), 2002, pp. 113-132.

Gutkin, T. B. "Collaborative versus directive/prescriptive/expert school-based consultation: Reviewing and resolving a false dichotomy", en Journal of School Psychology, 37(2), 1999, pp.161-190.

Hylander, I. "Conceptual change through consultee-centered consultation: A theoretical model", en Consulting Psychology Journal: Practice and Research, 64(1), 2012, 29.

Lago, J. R., y Onrubia, J. "Una estrategia general de asesoramiento para la mejora de la práctica educativa", en Profesorado. Revista de currículum y formación del profesorado, 12, 1, 2008, pp. 1-13

Lago, J. R., y Onrubia, J. Asesoramiento psicopedagógico y mejora de la práctica educativa, Barcelona, Horsori, 2011.

Luque, A. "¿Imprescindibles o desconectados? Sentimiento de competencia y necesidades de formación de los orienta- 
dores en los departamentos de orientación”, en C. Monereo y J. I. Pozo (Coords.), La práctica del asesoramiento educativo a examen, Barcelona Graó, 2005, pp. 221-240. Mauri, T. "La formación de los profesionales asesores: actualización y autoformación”, en C. Monereo y I. Solé (Eds.), El asesoramiento psicopedagógico: una perspectiva profesional y constructivista, Madrid, Alianza Psicología, 1996, pp. 479-499.

Monereo, C., y Solé, I. "El modelo de asesoramiento educacional-constructivo: dimensiones críticas”, en C. Monereo e I. Solé (Eds.), El asesoramiento psicopedagógico: una perspectiva profesional y constructivista, Madrid, Alianza Psicología, 1996, pp. 15-32.

Pérez Cabaní, M L., y Carretero, R. "Els sabers professionals de l'assessor psicopedagògic", en Ambits de psicopedagogia: Revista Catalana de Psicopedagogia I Educació, núm. 25, 2009, pp. 25-28.

Perrenoud, Ph. Desarrollar la práctica reflexiva en el oficio de enseñar: profesionalización y razón pedagógica, vol. 1, Barcelona, Graó, 2004.

Reschly, D. J. "A review of continuing education programs", en J. E. Zins, T. R. Kratochwill, y S. N. Ellicot (Eds.). Handbook of consultation services for children, San Francisco, Jossey Bass, 1993, pp. 351-372.

Rosenfield, S. "Best Practice in Instructional Consultation and Instructional Consultation Teams", en A. Thomas y J. Grimes (Eds.), Best Practices in School Psychology V, Bethesda, MD, National Association of School Psychologists (NASP), 2008, pp. 1645-1660.

Sánchez, E. "El asesoramiento psicopedagógico: un estudio observacional sobre las dificultades de los psicopedagogos para trabajar con los profesores", en Infancia y Aprendizaje, 91, 2000, pp. 5577.

Sánchez, E., y García, R. "Estrategias de colaboración, ayudar a ayudar”, en Marín, E. y Solé, I. (Coords), Orientación educativa, modelos y estrategias de intervención, Barcelona, Gráo, 2011, pp. 34-51.

Schulte, A. C., y Osborne, S. S. "When assumptive worlds collide: A review of definitions of collaboration in consul- 
tation”, en Journal of Educational and Psychological Consultation, 14(2), 2003, pp. 109-138.

Schön, D. El profesional reflexivo. Como piensan los profesionales cuando actúan, Barcelona, Paidós, 1998.

Showers, J., y Joyce, B. "The evolution of peer coaching", en Educational Leadership, 53(6), 1996, pp. 12-16.

Solé, I. Orientación educativa e intervención psicopedagógica, Barcelona, Horsori, 1998.

Solé, I. "Formación continua y competencia profesional de los asesores psicopedagógicos”, en C. Monereo y J. I. Pozo (Eds.), La práctica del asesoramiento educativo a examen Barcelona, Graó, 2005, pp. 273-286.

Solé, I. y Martín, E. "Un modelo educativo para la orientación y el asesoramiento", en E. Martín e I. Solé (Coords.), Orientación educativa: Modelos y estrategias de intervención, Barcelona, Graó, 2011.

Swafield, S. "Critical friendship, dialogue and learning, in the context of Leadership for Learning", en School Leadership and Management, 28(4), 2008, pp. 323-336.

Tapia, G. "Formación para el asesoramiento a las escuelas: Un proceso emergente en México", en Profesorado. Revista de curriculum y formación del profesorado, 12,1, 2008, pp. 1-15.

Thomson, Ch. "Collaborative consultation to promove inclusion: voices from the classroom", en International Journal of Inclusive Education, 17 (8), 2013, pp. 882-894.

Truscott, S. D., Kreskey, D., Bolling, M., Psimas, L., Graybill, E., Albritton, K., y Schwartz, A. Creating consultee change: A theory-based approach to learning and behavioral change processes in school-based consultation", en Consulting Psychology Journal: Practice and Research, 64(1), 2012, 63. Vélaz de Medrano, C., Manzanares Moya, A., López Martín, E. y Manzano Soto, N. "Competencias y formación de los orientadores escolares: estudio empírico en nueve comunidades autónomas", en Revista de Educación, Extraordinario, 2013, pp. 261-29.

West, J. F., y Cannon, G. S. "Essential collaborative consultation competencies for regular and special educators", en Journal of Learning Disabiliti, 21(1), 1988, pp. 56-63. 